in one case, in which three generations were affected. Huntingdon's chorea begins usually in the senile period of life, but its heredity is well-marked, and so is that of the peculiar rigidity called Thompson's disease, a lifelong peculiarity of action of the motor nerve structures, also called myotonia congenita.

I must pass some interesting diseases-paralysis agitans, tremors, Graves's disease, etc.-in which heredity can be traced seldom, but sometimes remarkably. I think it is probable that we shall hereafter find that special forms of heredity are associated with other characters in a manner that will make it an aid in distinguishing maladies now classed together, but for this we must wait.

A third class of diseases in which heredity is operative consists of the so-called functional affections. Some of them vary extremely in degree, and baffle attempts to group them so as to discover their relation to heredity. Such are hysteria and the defective nerve strength, which may manifest itself in varied ways, and which we are all drawn, sometimes against our better judgement, to designate " neurasthenia." Other functional disorders, such as chorea and neuralgia, have relations too complex for the clear discernment of heredity, and migraine, defined as it is, is connected with quite different constitutional states than disease of the nervous system. Two forms of functional disease have been much studied in relation to heredity - epilepsy and insanity.

Some cases of epilepsy are outside our subject. Those that result solely from organic lesions of the brain, from disease, or trauma, or difficult birth, are not concerned with heredity. The question of acquisition is here important. I confess I have always acted on the belief that the tendency to convulsions in such acquired cases, without other evidence of disposition, may be regarded as not transmissible. I have never hesitated, on this ground, to permit the marriage of a sufferer, and I have not had any evidence to show that I was wrong in doing so, however early in life the damage arose. I do not question the guinea-pigs of Brown-Séquard, but I do ques tion an inference from guinea-pigs to men. But such cases of "organic epilepsy" are rare. They constitute so small a proportion of the cases as to have little influence in statistics of heredity. The difficulties in ascertaining the facts of family histories are vast. Among hospital patients genuine ignorance often hinders. A woman brought her son for epilepsy and denied that any of his relations had had fits. As soon as she had left the room she returned with two of his brothers suffering from them. It had not occurred to her that a brother is a relation. In patients seen in private, the stigma supposed to attach to some diseases leads to concealment, which produces actual ignorance in another generation. A middle aged lady with an epileptic nephew denied any neurotic antecedents, and was indignant when $I$ told her that three great-aunts had been in asylums. But it was so.

In my own inquiries into the heredity of epilepsy I have limited myself to these two maladies, epilepsy and insanity. I find that in the cases seen in private there is a family history of one or both in 47 per cent., in antecedents and present or past collaterals. The difficulties I have just mentioned justify the assumption that at least 50 per cent., and probably more, really present a family tendency. Among hospital patients the number is not more.than 35 per cent., so great a difference does ignorance apparently make. In the facts presented the percentage was taken from 2,400 cases, of which 600 were seen in private. The proportions are much less than have been ascertained by others who have included a larger number of diseases. It may be well to confine myself to the cases in which a parent suffered. Jeremy Taylor tells us, in one of his sermons, that "an epileptick son doth often come from an epileptick father."

Of the total cases in which inheritance was paternal the father himself suffered in 40 per cent., when maternal the mother suffered in only 36 per cent. When epilepsy itself occurred in a parent, it was the father who was epileptic in 49 per cent. of the cases, the mother in 51. The cases with insanity in a parent are only one-third the number with parental epilepsy. Of the cases with parental insanity the father was insane in 37 per cent., the mother in 63. One effect of heredity is to increase the female cases. Where it was absent the excess of males amounted to 4 per cent., but in cases with heredity the same excess was presented by females. This is partly due to the fact that inheritance is more frequently from the mother's side, by 13 per cent., and that then females are in excess by 18 per cent.

The influence of heredity is sometimes strikingly emphasized by the number of sufferers in a family. In one instance six brothers suffered, their mother, two of her sisters, and both her parents. In another, five sisters, five of their children, the mother, her brother's son and her own mother.

Such facts might be multiplied almost indefinitely regarding many diseases. But we are told we are working on an entirely wrong method. Diem states that he bas found neuropathic heredity in nearly 70 per cent. of healthy persons. If he has included every conceivable malady that involves the nervous system it is quite credible, but of no significance. It has been urged that the essential facts for every member of each generation must be recorded to make observations of value.

But I may not take up your time longer, for there are here to.day those from whom we may learn, who have given up their ability and time to the scientific study of heredity. I hope that they will tell us how we may make our future observations of more service.

\section{TWO CASES OF UTERINE FIBROIDS SHOWING PERITHELIOMATOUS CHANGES : LONG IMMUNITY FROM RECURRENCE AFTER OPERATION.*}

\section{ALBAN H. G. DORAN, and CUTHBERT LOCKYER,} F.R.C.S.,

SENIOR SURGEON, SAMARITAN SENIOR PHYSICIAN TO OUT-PATIENTS, FREE HOSPITAI. SAMARITAN FREE HOSPITAL.

\author{
[Abstract. $]$
}

ThE following are the details of the cases:

CASE I.-Cystic Uterine Fibroid of large size in a 5-para aged 49: Infection of a loculus after tapping Supravaginal hysterectomy-removal of the fibromatous ovary: Opposite Fallopian tube and ovary not removed: Uterine tumour malignant (perithelioma) according to microscopic appearances:- Convalescence protracted through infection, probably from the tapped loculus: Speedy recurrence expected, probably fro: che tapped locins: Speedy thecurrence expected, after operation.

E. J., aged 49, was admitted into Mr. Doran's wards in the Samaritan Free Hospital in November, 1903. The abdomen had been enlarging for seven years. She had been tapped five weeks before admission, and over a pint of ropy fluid had come away without much diminution in the size of the tumour. The periods had recently been irregular. The patient had been married for thirty years, and had borne five children, the last confinement occurring eighteen years before admission.

On admission the patient looked fairly healthy, thin yet hardly On admission the patient looked fairly healthy, thin yethardy
cachectic. Both legs were slightly oedematous. The abdomen
was greatly distended, the measurements on November 5th was greatly distended, the measurements on November 5th being: Girth at umbilical level, $55 \frac{1}{2}$ in.; ensiform 71 in.; right umbilicus, $16 \frac{1}{2}$ in.; umbilicus to symphysis pubis, $7 \frac{1}{2} \mathrm{in}$.; right anterior superior iliac spine to umbilicus, $11 \frac{1}{3}$ in.; left ditto to umbilicus, $9 \frac{1}{2}$ in. The integuments of the abdominal wall were neither glossy nor oedematous, and there were no enlarged no high up in the epigastrium, and fluctuation at every point, not transmittible from flank to flank nor from the upper to the lower limits of the tumour. There was a scar below the umbilicus, the result of the recent tapping, and the parietes were much less tense at that point than higher up, as the above were much less tense at that point than higher up, as the above
measurements showed. This fact suggested loculi. The uterus measurements showed. This fact suggested loculi. The uterus no part of which came down below the brim of the pelvis.

\section{Operation.}

On December 1st, 1903, Mr. Doran removed the tumour. When the peritoneal cavity was opened a very vascular cyst wall was exposed. The omentum was strongly adherent, with its vessels greatly dilated. An artery and vein were ligatured, the remaining vessels were clamped, and the omentum detached and tied in segments. Four inches of the transverse colon, including the splenic flexure, were firmly adherent to the cyst wall, a thin layer of which was detached, leaving it on the bowel, which was then set free. The : under surface of the transverse mesocolon adhered firmly to the upper part of the tumour, and was torn in two places, but the lacerations were longitudinal and did not involve any of the big vessels. Several

* Read at a meeting of the Obstetrical and Gyneecological Section $*$ Read at a meeting of the Obstetrical and Gynar
of the Royal Society of Medicine, October 8th, 1908. 
hig loculi were broken down to make the extraction of the tumour easier. Unfortunately one loculus-that which had been tapped-was full of fetid pus.

The ovaries and Fallopian tubes were found to be separate from the tumour, which sprang from the uterus and from the roat of the right round ligament. A pint and a half of saline fluid were transfused under each breast; in the meantime the right ovarian vessels, a very big artery in the right round ligament, and some vessels in the left broad ligament were with the right made in front of the uterus during amputation was sewn over the raw surface of the uterine stump. Some large vessels in the raw surface of the uterine stump. Some large vessels in
the right broad ligament had to be secured. The two lacerathe right broad ligament had to be secured. The two lacera-
tions in the transverse mesocolon were repaired, and the edges tions in the transverse mesocolon were repaired, and the edges means of sutures. The peritoneal cavity was flushed with means of sutures. The peritoneal cavity was flushed with applied to the abdominal wound; several pints of saline fluid were left in the abdomen.

\section{Description of Parts Removed.}

Dr. Cuthbert Lockyer reported that the specimen, which weighed $15 \mathrm{lb}$., consisted of the uterus with its right appendages, the latter including a fibroma of the right ovary of the size of a swan's egg. The uterus was of the size of two fists. There was an interstitial fibroid in each of its lateral walls. From the left cornu sprang a large pedunculated fibroid outgrow the left cornu sprang a large pedunculated fibroid outof the fundus proceeded upwards a cystic fibroid growth of the same size as the enlarged uterus itself.

Under the microscope the large pedunculated tumour presented several areas of marked peritheliomatous change. Such areas were not detected in the other growths. Dr. Lockyer considered that the fact that no recurrence had taken place since the operation might be accounted for partly by the limited area of the change, many sections showing no sign of the new disease; partly by the pedunculation of the tumour, and partly by the fact that peritheliomata are less malignant than the more ordinary types of sarcoma.

\section{Convalescence.}

Convalescence was not rapid. On the eighth day an area of induration could be felt about the seat of the ligatured omentum. This area disappeared on the fifteenth day, when there was a very free discharge from the rectum of a greasy fluid with old clots. Apparently one of the ligatures on the omental vessels, clots. Apparently one of the ligatures on the omental vessels, or one of the sutures applied to the piece of cyst wall on the
bowel, had become infected, either from the intestine or from the pus which escaped from the loculus which had been tapped before the operation.

By April, 1906, the patient had grown very corpulent, weighing over $17 \mathrm{st}$. The menopause was not complete antil early in 1907. Considering that the patient was subject to slight mental disturbance, Mr. Doran thought that the removal of both ovaries would have been unjustifiable, the left being sound.

On August 5th, 1908, she was in very good health. The abdominal walls had become extremely fat and pendulous ; the cicatrix of the abdominal incision was free from hernia or new growth. The cervix was small and fairly movable. There was no trace of any new growth or inflammatory deposit in the pelvis or abdomen.

CASE II.-Cystic Uterine Fibroid invading the left broad ligament and sigmoid mesocolon in a 2-para, aged 36-marked changes in left Fallopian tube and ovary: Deposit in parametrium, of doubtful nature: Uterus amputated above os externum, total hysterectomy appearing impracticable: Uterine tumour and growths in left tube and ovary malignant (perithelioma) according to microscopic appearances: Speedy recurrence expected, yet patient living and well two years and seven months after the operation.

A. F., aged 36, was admitted into Mr. Doran's wards in the Samaritan Free Hospital at the end of May, 1905. Fifteen months previously she had discovered a swelling in the left side of the abdomen, which had recently grown quickly.

The patient looked very healthy, and might have passed for a woman of 30 years of age. She had borne two children, both in robust health. Some injury seemed to have occurred during robust health. Some injury seemed to

On admission the abdomen was seen to be irregularly distended by a tumour which lay more to the left of the middle line than to the right, and was deeply lobulated. A soft lobe on the right reached above the umbilicus, a still softer lobe, partly resonant on percussion, made up the entire left portion, a firm lobe lay in the right iliac fossa, and a small, very hard body could be defined immediately above the pubes, almost in the middle line. The entire tumour was movable to a limited extent. The cervix was very firm in consistence and continuous with the hard lobe above the pubes. A suspicious irregular bleeding growth was detected on the vaginal wall in the left fornix. It probably was a result of some injury during the seoond labour. At the time of admission it seemed to be a perforated the vaginal wall. The patient had quite recently perforated the vaginal wall. The
suffered from severe menorrhagia.

\section{Operation:}

Mr. Doran operated on June 17th, 1905. The omentum was strongly adherent to the surface of the big left lobe. The uterus, very bulky, formed the hard lobe which lay above the pubes. The left Fallopian tube was very long and thick; the left ovary was somewhat enlarged and closely applied to the big left lobe, which had opened up not only the left broad ligament, but also the sigmoid mesocolon, so that the sigmoid colon ran down the surface of the big lobe towards the resceribed. tion of the lobe, the base of which lay very deep, proved difficult. The colon and its mesentery were detached without damage to either. The left ovarian vessels were tied and damage to either. The left ovarian vessels were tied and divided. The whole tumour was thus set free, except from its parts adjacent to the tumour in the left iliac region seemed parts adjacent to the tumour in the left iliac region seemed highly unfavourable. There was deposit in the left parametrium, between the cervix and the bladder, and in the left round liga-
ment, which was very thick. The right ovarian vessels were tied off, the broad and round ligaments cut through, secured and divided, and the uterus amputated above the cervix, which was fixed by the deposit. The uterine vessels were secured as they were divided; they lay in very dense tissue, and could not be isolated before ligature in the usual manner. The muscular walls of the uterus appeared very unhealthy, and a good peritoneal flap could not be the uterine walls above the cervix, and the raw surfaces were sewn together with No. 1 silk in two layers. The omentum, full of very big vessels, was resected close to the transverse colon, as oi very big vessels, was resected close to the transverse colon, as
it was unavoidably damaged when separated from its close it was unavoidably damaged when separated from its close iliac fossa; the peritoneum was flushed with saline fluid, and, as the pulse was failing, several ounces of the same fluid, with 30 minims of adrenalin, were injected into the right median basilic vein, and liquor strychninae was administered hypodermically. The abdominal walls were closed in two layers.

The tumour, with the body of the uterus, weighed $2 \frac{1}{2} \mathrm{lb}$. During the enucleation of the big left lobe from the iliac fossa, about a pint of pale yellow fluid escaped from a cystic cavity in its interior. Small solid masses, suggesting myosarcoma, grew connective tissue.

Description of Parts Removed.

Dr. Cuthbert Lockyer reported that the parts removed were made up of a thick-walled uterus, both appendages and a large. adherent, partly cystic uterine growth which had burrowed deeply into the left broad ligament. The uterus had not been removed entire, so that its cut surface presented a very broad base. Its walls showed on section numerous small, smooth, pea-like growths. The left appendages were extensively altered. The Fallopian tube was 6 in. long and 1 in. in diameter and was thickened and nodular. The mesosalpinx was made
thick by a nodular, solid growth which fused with the large thick by a nodular, solid growth which fused with the large
partly solid and partly cystic growth extending into the parapartly solid and partly cystic growth extending into the parametrium from the left side of the uterus. This growth measured
12 in. by 8 in. by 4 in. in its three diameters. Its lower half was $12 \mathrm{in.}$ by $8 \mathrm{in}$. by $4 \mathrm{in}$. in its three diameters. Its lower half was
cystic and its upper part solid. The cystic portion was rough cystic and its upper part solid. The cystic portion was rough and devoid of peritoneum, whilst the upper part was covered by and contained a nodular growth similar to that which invaded the tube and mesosalpinx. Microscopic sections were prepared from the uterine wall, the left Fallopian tube, the mesosalpinx, the left ovary, the large tumour in the left parametrium, and and tissues to be invaded by a typical perithelioma.

In this, as in the former case, there was not that speedy uncomplicated convalescence so usual after hysterectomy for fibroid. On July 23rd the evening temperature rose to $103^{\circ}$, and reached that height every evening until the $29 \mathrm{th}$. This symptom was associated with fetid stools, followed by fetid discharge independent of the motions. Parametritic inflammation had probably set in, yet it was strange that no suppuration occurred, nor did any characteristic infiltrations develop in the groin and above the vaginal fornices. Mr. Doran ascribed the symptoms to a much more serious condition, and never expected to hear satisfactory news of the patient after her discharge from the Samaritan Hospital at the beginning of August, 1905. To his great surprise, the patient came to see him at the hospital on November 2nd, 1905, and looked as well as she did before the operation. She complained of attacks of hot flushings and trembling about three times a week, and the periods had never been seen. The abdominal wound was well healed, and there was no deposit to be felt in the parietes nor any tumour to be defined behind them. The cervix was fixed; there was no trace of the bleeding growth on the vaginal mucosa in the left fornix noted before the operation, but simply a distinct thickening of the vagina bearing two small fleshy wattles. Thus at least there had been no exfension of the suspected malignant disease into the vagina. Ovarian tabloids were prescribed to relieve the menopause symptoms. About a year later $\mathbf{M r}$. 
Doran received a false report of the patient's death, but on February 12th, 1908, a patient came to see him at the hospital. She complained of dyspepsia, due, it appeared, to excessive tea-drinking, but she added that she had gone on wonderfully well since her operation. Then, on looking into his notebook, Mr. Doran found she was Case II, and there was a report of her death. On further inquiry it transpired that the report referred to some other patient with a similar name and complaint, who had never been under his care. The patient looked very healthy, just as she appeared before the operation, and had distinctly gained flesh. There had been no period since the operation, and the menopause symptoms had passed away soon after her last visit in 1905. On examination, the abdominal cicatrix was found to be perfectly healthy, and the parietes supple. There was no evidence of the existence of any abdominal tumour. The cervix had become very small, and was still fixed, the two fleshy wattles in the left fornix had almost disappeared. There was a little resistance above the left fornix just definable on bimanual palpation. The right and posterior fornices were free. Such was the patient's condition two years and seven months after the operation. Mr. Doran declared that in the whole of his operative experience he had never come across a case where the after-history so thoroughly belied operative and pathological evidence.

These cases show the imperative necessity for careful observation of all patients from whom uterine fibroids have been removed. Only by such observation can we expect to gain anything like accurate knowledge as to the effects on the patient's organism of the removal of the fibroid uterus with or without cervical or ovarian tissue, so that we may see how far clinical experience agrees with the physiological experience of Blair Bell, Bond, Carmichael and Marshall, and others; and only by such observation can we expect to calculate with something like precision the chances of malignant, or apparently malignant, changes being overlooked or misinterpreted.

\section{SOLID FIBROID OF OVARY OBSTRUCTING DELIVERY.}

By J. STEWART, M.B., M.Сн., M.A.O.,

$$
\text { LEEDs. }
$$

THE followisg case occurred in a lady married two years, and who was attended by me in her first confinement.

Before her marriage the patient, who was aged 26, had complained at times that she felt something roll about in her abdomen, this being accompanied by sudden faintness and sickness. No opinion could be formed at the time as to the cause of this, but subsequent events made things clear. When called to see her in labour, it was found that there had been pains for two hours. On making a vaginal examination the pains for two hours. On making a vaginal examination the quisitely sensitive that it immediately flashed through my mind, without any attempt at a differential diagnosis, that I mas dealing with an ovarian tumour which was being comwas dealing with an ovarian tumour which was being com-
pressed. This smooth tender mass was opposite the right pressed. This smooth tender mass was opposite the right pelvis and the head of the fetus, advancing in front of the latter. The cervix could be felt high up and just within the true pelvis. It was soft, dilatable, and about the size of half a crown. Rectal examination assured me that the mass was not faecal, although that was negatived by the great tenderness present.

Under chloroform the hand was passed into the vagina, the uterus and fetal head pushed to one side, when the tumour gently slid back into the abdomen. With the hand still in the gagina the cervix was dilated, forceps applied, and child vagina the cervix was dilated, forceps applied, and child
delivered. The puerperium was uneventful. Six weeks afterwards I removed a solid fibrous tumour of the ovary, somewhat irregular in shape, and about the size of an orange. It was very irregular in shape, and about the size of an orange. It was very easy to remove, there being no adhesions, and a sufficiently long was without interest. The train of symptoms mentioned at the beginning, of something rolling about with faintness and sickness; have entirely disappeared.

Of all forms of pelvic obstruction the most interesting and by far the most frequent is obstruction caused by an ovarian tumour. McKerron gives the frequency of ovarian tumour complicating pregnancy as about 1 in 2,500. The mortality is appalling when Nature is allowed to take its course, the maternal being about 25 per cent. and the infantile nearly 50 per cent. The question of treatment is debatable, and in pursuing the course I did I am afraid ran counter to the best English opinions. Bland-Sutton says:

When an ovarian tumour is discovered during labour and it impedes delivery, ovariotomy should be performed.

McKerron says :

Ovariotomy is the best treatment, and should be preferred when the patient can be placed under an experienced operator. When time and place does not admit of ovariotomy try reposition.

On the Continent there are two schools-one advocates Caesarean section, and this has the greater following; the other, ovariotomy.

On one point, however, every authority is agreed-never to attempt delivery until the tumour is removed either by reposition, puncture, or ovariotomy. The objections to ovariotomy are (1) great length of incision, because, as a rule, the surgeon has to get the uterus outside the abdomen to get at the tumour; consequently there is (2) great risk to the life of the fetus; (3) the surgeon must return the pregnant uterus, and sew up the abdomen over it.

Puncture is only mentioned to be condemned; it could have been of no use in the case of solid tumour reported here. There is just one possible exception, where the head is well engaged in the pelvis, and there is a cystic tumour low down in front of the head. In such a case puncture, and perform ovariotomy after delivery.

There are two objections to reposition. In pushing back the tumour, if a cyst it may rupture, or the pedicle may become twisted, and, as a rule, a long pedicle is present. As to the first, there is not much risk if gentleness be used. Instead of pushing । at the tumour, push up the head of the fetus and the uterus out of the way. As to the second, axial rotation with twisted pedicle, it will be announced by great pain, tenderness and vomiting, accelerated pulse, and rise of temperature. If these should supervene in a case in which reposition had been performed, there should be no hesitation about opening the abdomen. Personally, I would much prefer to open the abdomen after the uterus had been emptied, and to perform ovariotomy, than to do it when the abdomen was crowded with a pregnant uterus.

It is almost too much to ask of human nature not to try and replace, if it can be easily accomplished. By doing so, at the same time emptying the uterus, a difficult situation has been surmounted, leaving the tumour to be dealt with in the near future.

If the tumour cannot be pushed up from the pelvis into the abdomen, then, in spite of the teaching of Mr. BlandSutton (and there is no authority whose opinion would carry greater weight), I should not hesitate to perform Caesarean section, at the same time removing the tumour after closing the uterine incision; as these tumours have long pedicles, it would add hardly anything to the operation. By so doing the necessity of an incision from ensiform cartilage to pubis is obviated, the life of the fetus through eventration of uterus is not endangered, and the difficulty of closing the abdomen over a fully distended organ is done away with.

\section{A NOTE ON ABDOMINAL HYSTERECTOMY FOR CANCER OF THE CERVIX, AND ITS IMMEDIATE MORTALITY;}

\author{
WITH AN ANALYSIS OF SEVENTEEN CASES.
}

By T. BONHÔTE HENDERSON, M.A., M.B.Oxon., F.R.C.S.,

SURGical REgistrar to THE CANCER Hospital.

THE value of statistics of surgical operations is undoubted, yet they are often misleading owing to the predominant influence of the personal factor-the individual skill and experience of the operator. More especially is this true of any modern and extensive operation such as Wertheim has introduced to the profession, and which not many English surgeons have had the opportunity of performing in any large series of cases.

In a recent instructive article by Messrs. Berkeley and Bonney, ${ }^{1}$ a table was given of 18 cases with 3 deaths (mortality 16.6 per cent.), a result of which these authors 